\title{
Research Square \\ Photocontrollable Crystallization at the Topological Defect of a Liquid Crystalline Droplet
}

\author{
Kenji Katayama ( $\nabla$ kkata@kc.chuo-u.ac.jp ) \\ Chuo University https://orcid.org/0000-0003-3278-6485

\section{Yota Sakai} \\ Chuo University
}

\section{Article}

Keywords: Liquid crystalline droplet, Crystallization, Photo-induced phenomena

Posted Date: March 2nd, 2021

DOl: https://doi.org/10.21203/rs.3.rs-244385/v1

License: (c) (i) This work is licensed under a Creative Commons Attribution 4.0 International License. Read Full License 


\section{Photocontrollable Crystallization at the Topological Defect of a 2 Liquid Crystalline Droplet}

3 Yota Sakai $^{1}$ and Kenji Katayama ${ }^{1}$

41 Department of Applied Chemistry, Chuo University, Tokyo 112-8551, Japan;

$5 \quad *$ Corresponding author:

$6 \quad$ K. Katayama, Phone: +81-3-3817-1913, E-mail: kkata@kc.chuo-u.ac.jp

\section{Abstract}

Photo-controllable crystallization at the topological defect in an LC droplet was demonstrated.

10 The dye molecules dissolved in a surfactant solution outside the LC droplet were promoted to move

11 into the droplet by the light absorption. Nuclei emerged tens of seconds after light irradiation and

12 moved toward the topological defect located at the droplet center, forming a branch-shaped crystal.

13 This phenomenon was reproduced for three different dyes, and photo-induced migration, nucleation, and crystal formation were discussed as a possible mechanism.

16 Keywords: Liquid crystalline droplet; Crystallization, Photo-induced phenomena 


\section{Introduction}

19 Crystallization of chemicals is a fundamental process for materials from the viewpoints of basic

20 science and practical applications. In a well-established theory by La Mer, ${ }^{1}$ an over-saturated solution

21 starts to provide nuclei of a crystal at the initial stage, and subsequently, the nuclei grow in size as

22 long as the concentration of the chemical is over-saturated. For practical purposes, crystallization is

necessary for the pharmaceutical industry, and also it is vital in basic science for the determination of

the molecular structure of chemicals and proteins by x-ray diffraction analysis (XRD). However, many

chemicals cannot be crystallized due to fundamental reasons, such as solubility, chemical interaction,

impurities, a short amount of chemicals, etc. Much effort has been made for crystallization by

preparing over-saturated concentration via temperature, stirring speed, $\mathrm{pH}$, etc, ${ }^{2}$ and the technological

progress has continued. For example, flow-based crystallization has been developed by control of anti-

solvent and introduction of plug flow. ${ }^{3-5}$ A unique technique, where the porous metal-organic

framework has been demonstrated to absorb guest molecules and orient them in a crystalline form

called as 'crystalline sponge method.' 6,7

The liquid crystal (LC) is a phase between the solid and liquid, where it has a periodic structure

in a specific direction like a crystal and has fluidity in another direction like a liquid, and this phase 
purposes in our daily life. These days, topological defects in LCs have been paid much attention to

37 because they could potentially control the structure and motion of LCs. The topological defect is an

38 orientationally disordered point of LC molecules, where the molecular orientation cannot be defined.

39 Intentionally formed topological defects by the photo-alignment layer could control the alignment of

40 LCs and can be utilized for various thin optics. ${ }^{9-11}$ When a droplet is formed with LC, different types

41 of topological defects were formed inside and on the surface. ${ }^{12}$ These droplets and spheres have been

42 studied intensively as an 'active matter,' where the object could move around spontaneously like a

43 living object by external energy sources. The motion of LC spheres was controlled by several

44 topological defects, which also caused the self-assembly of molecules. ${ }^{13}$ Topological defects were

45 further investigated in biology because it was found that biological cells aligned like LC molecules

46 and had topological defects. Furthermore, they could control the collective motion and biological

activity depending on the types of defects. ${ }^{14,15}$

The defects in LCs sometimes could help to align and assemble colloids and molecules.

49 Amphiphilic molecules were self-assembled to form nanostructures at the line defect (disclination). ${ }^{13}$

50 LC ordering could align gold nanorods to the alignment direction in a lyotropic $\mathrm{LC},{ }^{16}$ and also gold nanoparticles were concentrated at the dislocation of the smectic LC. ${ }^{17,18}$ The force induced by the observed for dense colloid particles in LCs, ${ }^{20}$, and the disclination was intentionally controlled to make 

category of active matters consisted of LCs, which could move around in a surfactant solution due to an induced convective flow inside and outside the droplets while gradually dissolving into it. ${ }^{22-24} \mathrm{We}$ focused on the photo-controlled motion of the LC droplets by promoting adsorption and desorption of molecules at the interface and could demonstrate the LC droplets approaching to and drawing away from a light source, ${ }^{25,26}$ and showed a clockwise/anti-clockwise rotation under the light. ${ }^{27}$ During this study, we found a crystallization of a chemical was triggered by light at the topological defect in a pure LC droplet, even though the chemical was dissolved outside the LC droplet. Under the light irradiation whose wavelength matches the absorption of chemicals, a crystal was formed inside an LC droplet. It grew at the center of the droplet (topological defect) with a branched shape like an ice crystal. This is the first demonstration of the topology-induced crystallization under the geometrical frustration. ${ }^{28}$ In this paper, we will show the demonstrations for the formation of several crystals and describe the possible mechanism of this phenomenon.

\section{Result and discussions}

Figure 1 shows an image sequence of a 5CB droplet surrounded by an SDS solution with $p$ - 
at the center, which is determined by the $5 \mathrm{CB}$ molecular alignment dominated by the boundary

condition of the $5 \mathrm{CB}$ and the SDS solution (homeotropic alignment). ${ }^{29}$ Surprisingly, we could recognize that small objects $(\sim 2 \mu \mathrm{m})$ started to nucleate inside the droplet about $30 \mathrm{~s}$ (Fig. 1(c)), and getting larger (Fig. 1(d)-(f)). Simultaneously, these objects were drawn into the center of the droplet, namely to the topological defect. The objects were gradually connected to grow and shaped branched structures. When the light was turned off, the small nucleated objects ceased to form gradually. These

to the original branches to make additional branches (Movie S1 in Supporting Information (SI)).

(a)

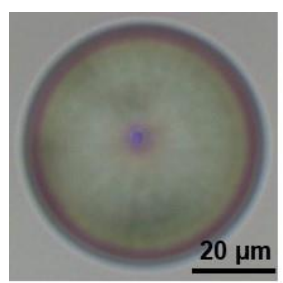

(f)

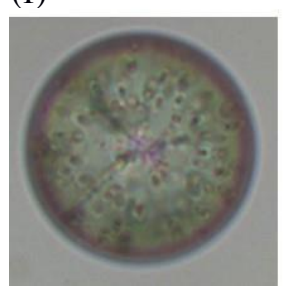

(b)

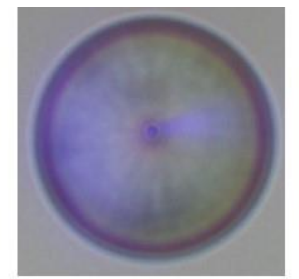

(g)

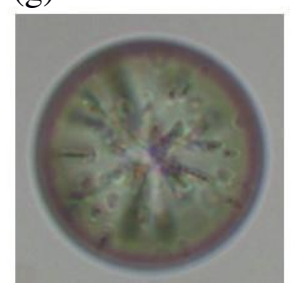

(c)

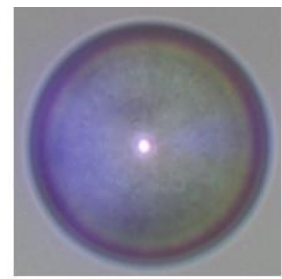

(h)

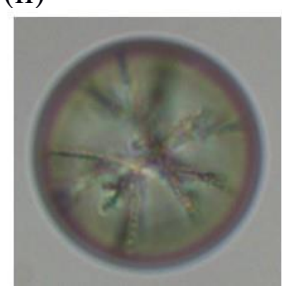

(d)

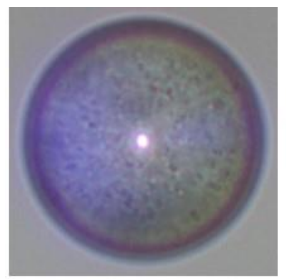

(i)

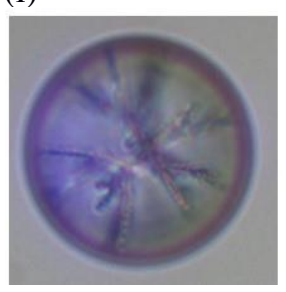

(e)

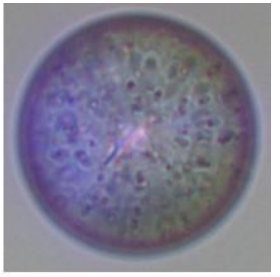

(j)

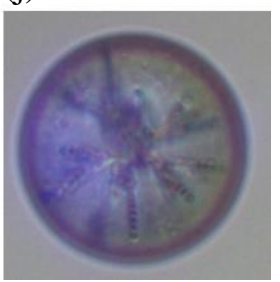

Fig.1 The snapshots of a 5CB droplet in an SDS solution with $p$-nitrophenol (0.01 wt\%) under the on-off operation of the UV light is shown; (a) before irradiation, (b) - (e) 0, 30, 60 and $90 \mathrm{~s}$ after the UV was turned on, (f) - (h) 0, 60, and $120 \mathrm{~s}$ after the UV light was turned off, (i) and (j) 0 and $30 \mathrm{~s}$ after the UV light was turned on again. 
of the 5CB droplets before and after the nucleation and growth by irradiation of the UV light, characteristics, suggesting the ordered alignment of molecules because the same color indicates the polarizer or analyzer direction in the black region, while they are in-between in the white regions. This were aligned in particular directions.
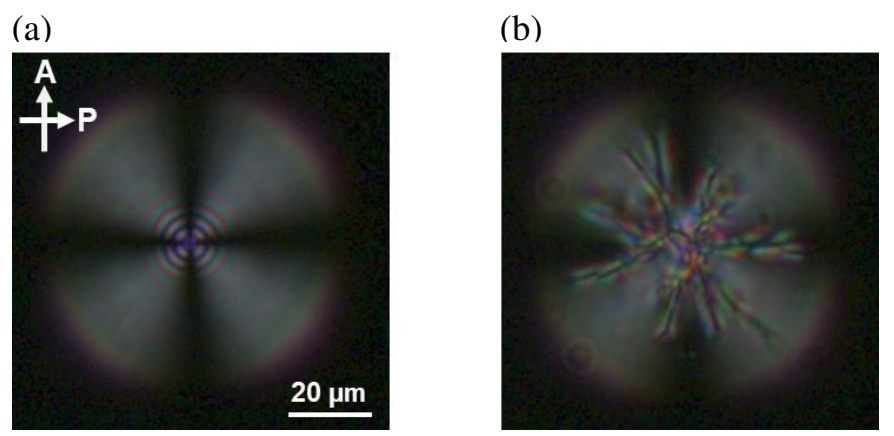

Fig.2 The snapshots of the 5CB droplet in an SDS solution with p-nitrophenol (0.01\%) observed by the polarization microscope under the crossed Nicole condition; (a) before irradiation (b) after the UV irradiation for $60 \mathrm{~s}$. The white arrow in (a) indicates the direction of the analyzer and the polarizer. 

only difference was the formation speed of the branched structure. The necessary condition for these

(a)
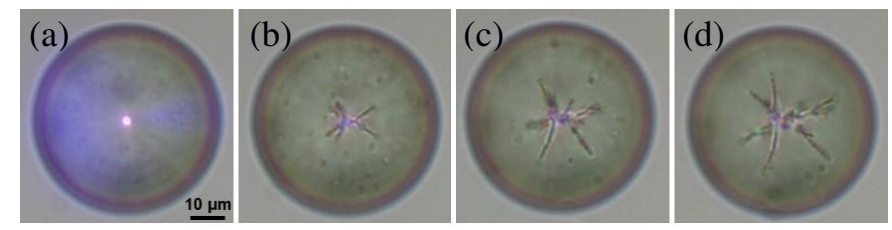

(b)
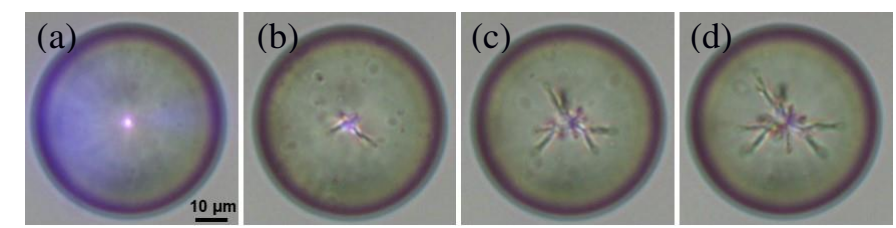

Fig.3 The snapshots of the 5CB droplet in an SDS solution including (a) alizari yellow GG and (b) chrome yellow under the on-off operation of the UV light is shown; (a) $120 \mathrm{~s}$ after the UV irradiation, (b), (c), and (d) correspond to 120, 240 and $480 \mathrm{~s}$ after the UV was turned on.

Raman microscopy was used for the characterization of the photo-generated crystals inside the

LC droplet. The crystal inside the 5CB droplet was measured under the same experimental conditions 
119 including the photo-generated crystal, which was assigned as $p$-nitrophenol. The Raman spectrum was

120 not obtained for $p$-nitrophenol solved in an SDS solution under the same experimental condition. At

121 this moment, we could not exclude the possibility of the formation of co-crystal of 5CB and $p$ -

122 nitrophenol, ${ }^{31}$ but we suppose that it is low considering this could happen for several different chemicals.

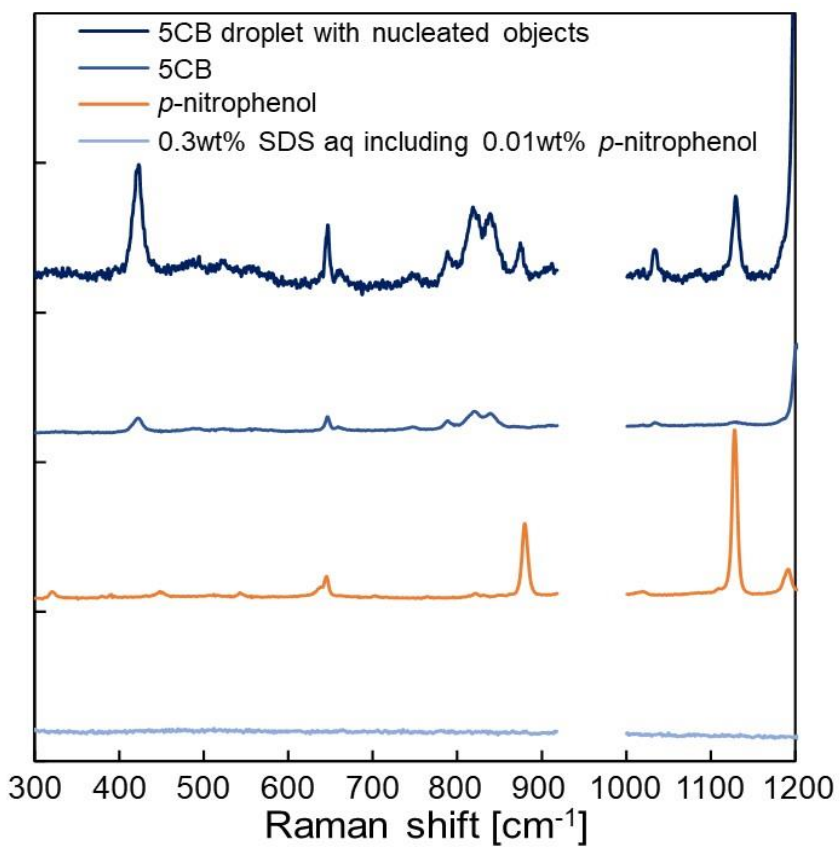

125 Fig.4 The Raman spectrum of a photo-generated crystal in a 5CB droplet (top). The Raman spectra 126 for pure $5 \mathrm{CB}, p$-nitrophenol (powder), and a $0.3 \mathrm{wt} \%$ SDS solution with $0.01 \mathrm{wt} \%$-nitrophenol are 127 shown for comparison. The spectral region from $920-1000 \mathrm{~cm}^{-1}$ was removed due to the noise of the 128 excitation light source. 
131 solution. The temporal change of the UV/Vis absorption spectrum of the outer solution was measured

132 during the UV irradiation to investigate the chemical resource. A single 5CB droplet with a volume of

$13320 \mu \mathrm{L}$ was prepared in a $0.3 \mathrm{wt} \%$ SDS solution with $0.001 \mathrm{wt} \%$-nitrophenol $(10 \mathrm{~mL})$ in a vial. The

134 outer SDS solution was sampled with $0.5 \mathrm{~mL}$ every 30 seconds during the UV irradiation, and the

135 absorbance of each sample was measured by UV/Vis spectrometer.

137 The absorption peak at $316 \mathrm{~nm}$ for $p$-nitrophenol (Fig.S2) gradually decreased during the UV

138 irradiation. This result indicates that the amount of $p$-nitrophenol in the outer SDS solution was

139 decreased during the light irradiation. This result supports that the dyes migrated from the outer

140 solution into the LC droplet, which was the source of the nucleated crystal.

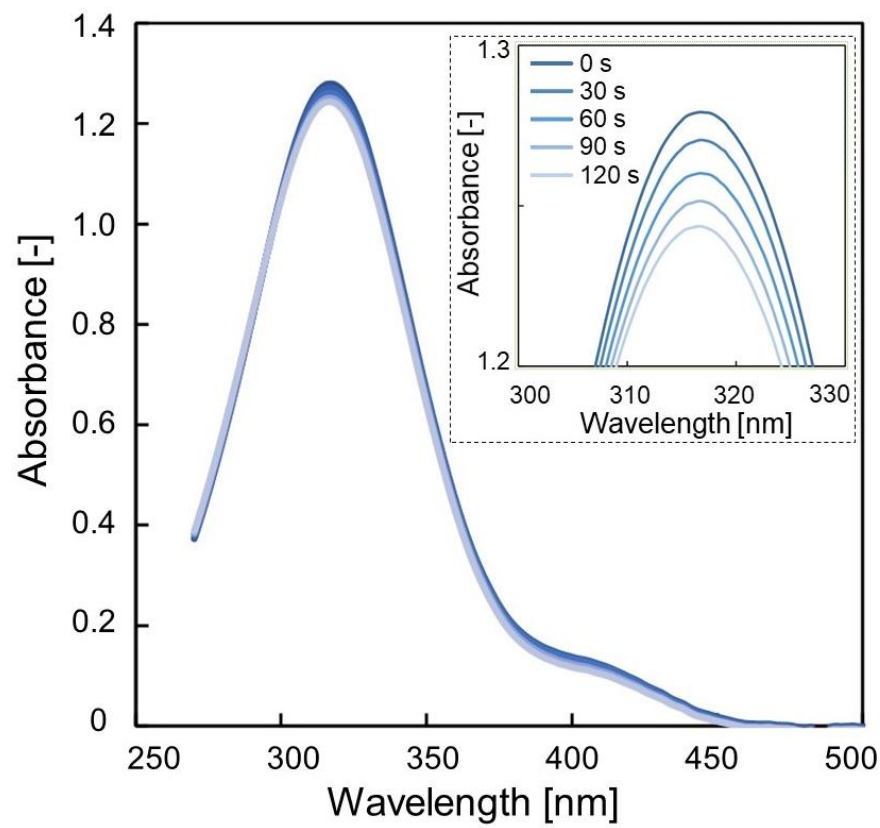

141 Fig.5 The temporal change of the UV/Vis absorption spectra for the outside SDS solution with $p$ 142 nitrophenol $(0.001 \%)$, including an 5CB droplet during the UV light irradiation. The outside solution was sampled every 30 second during the UV light irradiation. 

the exchange of the dye molecules at the interface between the LC droplet and the SDS solution. The drop measurement for the LC droplet in an SDS solution with $p$-nitrophenol (Fig.6). The UV light was irradiated for 20 seconds twice. The interfacial tension gradually increased during the UV irradiation and decreased after being turned off. This result indicates the desorption of molecules from the of the dyes initially adsorbed at the LC/water interface into the LC phase.

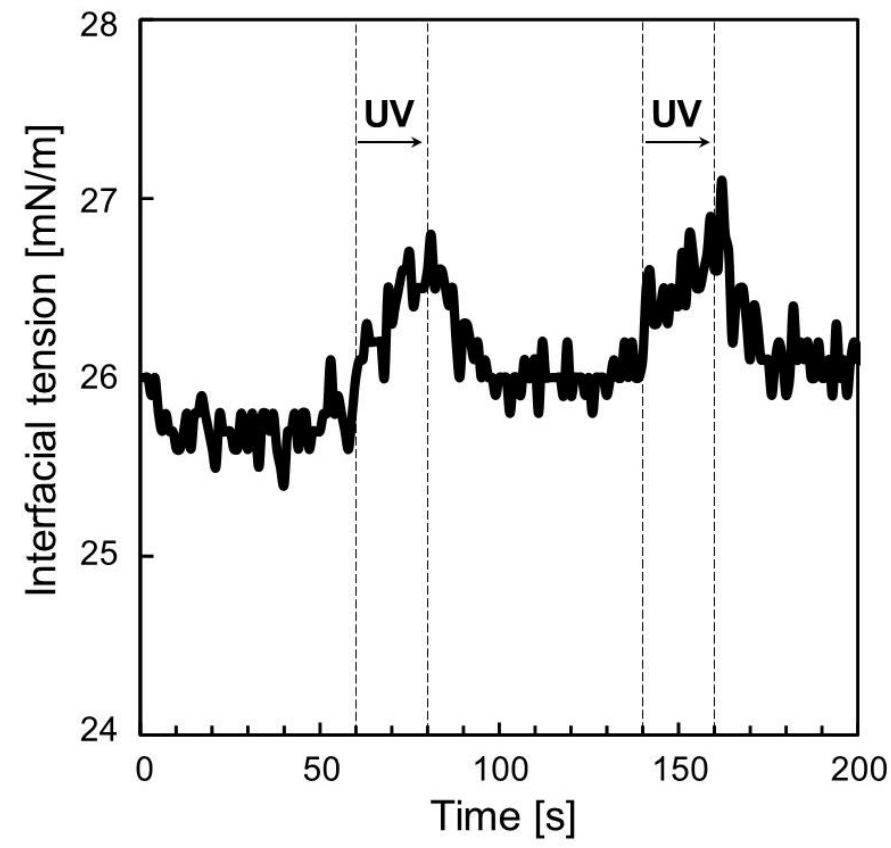

Fig.6 The change of the interfacial tension during the on-off operation of the UV light for a 5CB 154 droplet in an SDS solution with $p$-nitrophenol (0.01 wt \%). The UV light was irradiated twice for 20 seconds at 60 and 140 seconds during the measurement. 

interface by light, which indicates that the dyes were initially adsorbed at the LC/water interface. The contact angle of an LC droplet on the dye concentration in the outer solution was studied to verify it. A drop of 5CB with a volume of $20 \mu \mathrm{L}$ was dropped onto a hydrophobically-treated petri-dish. The contact angles of the 5CB droplets surrounded by $0.3 \mathrm{wt} \%$ SDS solutions $(0,0.1$, and $0.5 \mathrm{wt} \% \mathrm{p}$ nitrophenol) were measured. The pictures of each droplet are shown in Fig.S1 in SI. The contact angle decreased as the dye concentration in the outer solution increased. This result indicates the dyes adsorb at the interface in a static state, supporting our assumption. molecules dissolved in an aqueous surfactant solution are usually solubilized into the organic phase as a reverse micelle. The SDS molecules could make a reverse micelle of the dyes and promote solubilization into the LC phase. The effect of the reverse micellar solubilization was studied by changing the type of the surfactants. Instead of SDS, we used polyvinyl alcohol (PVA) as a protecting agent for the LC droplet. The PVA could keep the stability of the LC droplet interface by a random coil formation but does not make a reverse micelle for the dyes. ${ }^{29} \mathrm{~A} 5 \mathrm{CB}$ droplet was prepared in a PVA solution (1 wt $\%$ ) with $0.01 \mathrm{wt} \%$-nitrophenol, and the UV light was illuminated similarly. The result is shown in Fig. 7 (Movie S4 in SI). The nucleation of small objects started inside the LC droplet 
174 about $30 \mathrm{~s}$ after the UV light irradiation, which was similar to the SDS solution. This result indicates

177
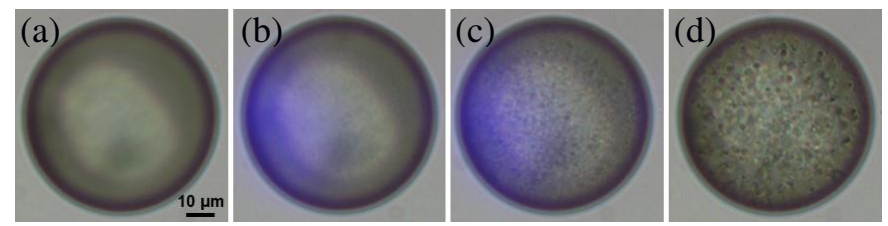

Fig.7 The snapshots of the 5CB droplet in a PVA solution with $p$-nitrophenol (0.01 wt $\%)$ under the UV on-off operation is shown. (a) before UV irradiation (b) and (c) $30 \mathrm{~s}, 90 \mathrm{~s}$ after the UV light turned on, (d) $60 \mathrm{~s}$ after the UV light turned off.

From this experiment, we had more important information on this crystallization process. As

we could confirm from Movie S4 and Fig.7, the small nuclei did not approach the droplet center, even

though the dye nucleation was induced in a PVA solution, too. The nucleated and grown small objects

kept fluctuating inside the LC droplet. Since PVA imposes the planer orientation of the LC molecules

(parallel) at the LC/solution interface, the LC alignment in the droplet becomes bipolar configuration, droplet has a crucial role in the build-up of the crystalline phase. 
192 No reactions proceeded during the light irradiation. This result indicates that the LC phase was

193 necessary for taking up the dyes into the LC phase or for the nucleation itself. Furthermore, we

194 investigated the temperature influence for the crystallization because the photo-absorbed dyes release

195 heat via photothermal relaxation and/or photo-isomerization in the case of azo-dyes. The behavior of

196 the 5CB droplet in an SDS solution with $p$-nitrophenol was observed under the temperature variation

197 in a temperature-controlled vessel. The sample temperature was initially set at room temperature

$198\left(25^{\circ} \mathrm{C}\right)$, and it was raised by $5{ }^{\circ} \mathrm{C}$, which is sufficiently higher than the calculated temperature rise due

199 to the photo-absorption of $p$-nitrophenol $\left(\sim 0.8^{\circ} \mathrm{C}\right)$, and was lowered to the room temperature again.

200 (Movie S6 in SI). No reaction was observed, and only the focus point was defocused under the

201 temperature variation. This result indicates that photo-excitation has a role in the crystallization

202 process, possibly for being taken up into the LC phase.

Based on the observations and considerations, we propose the following possible

crystallization mechanism. It is supposed that a part of dye molecules was initially adsorbed at the

205 LC/water interface, confirmed from the interfacial tension dependence on the dye concentration.

206 Based on the dynamic interfacial tension measurement, the desorption of these dyes into the LC phase

was promoted during the UV light irradiation. Also, the dyes could be provided from the outside 
210 one of the possibilities is that the photo-induced dipole (excited state) prefers the molecularly oriented

211 environment like LC. This intake could be a similar process as the gold nanorods with a large dipole

212 moment were taken and aligned by LCs to show a long-range ordering. ${ }^{16}$ This should be studied in

213 more detail on the molecular properties of dyes and LCs based on the molecular dynamics.

214 Overcoming the saturation concentration of the dyes, they start to form nuclei at random positions.

215 Then, the nuclei gathered to the topological defect at the center and grew with a branched shape. The

216 accumulation of objects to the LC defects (dislocation) was previously observed, ${ }^{13,17,18}$ where a

217 preferable environment for assembly of objects is provided by reducing the high free energy core of

218 the LC defects. ${ }^{13}$ In our case, the nuclei were collected at the point defect at the center. The object can

219 keep the crystalline phase confirmed by the crossed-Nicole observation, and this suggests that the LC

220 provided a preferable environment for molecular orientation suitable for the crystal growth. It was

221 reported for pure LC molecules that the LC phase could work as a metastable state, inducing frustration

222 for the crystal formation. The LC phase could potentially ease the nucleation of molecules by the

223 ordering environment for extraneous molecules, ${ }^{28}$ and it would be a similar concept to the crystal sponge. ${ }^{6}$

\section{Conclusion}

We found a photo-controllable unique crystallization process at the topological defect of an 
228 LC droplet in a surfactant solution with dye molecules. The dye molecules, initially adsorbed at the

229 interface of the droplet/surfactant solution, were desorbed into the LC droplet during the light

230 irradiation. The dye molecules overcame the saturated concentration inside the LC droplet, starting to

231 form crystal nuclei at random positions. These crystals gathered to the topological defect, growing

232 with a branched shape in a crystalline form. This process was demonstrated for several different dyes

233 with the light absorption matching the irradiated light source. This is a brand-new crystallization

234 technique, and controllable by light, and also showed another interesting property of topological

235 defects. This methodology is a simple and easy method for crystallization and could crystallize various

236 molecules for pharmaceutical purposes and structural analyses for biomolecules.

238 Methods

239 LC droplets were prepared using a microfluidic device. A schematic drawing of the device is

240 shown in Fig. S2 in SI, and the detailed method was described. The typical size of the droplets was 50

$241 \mu \mathrm{m}$ in diameter. 

outer fluid. The solution included water-soluble dyes whose absorption wavelengths have an overlap

UV light illumination from the top side.

(a)

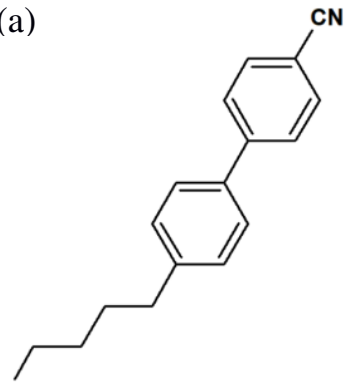

(c)

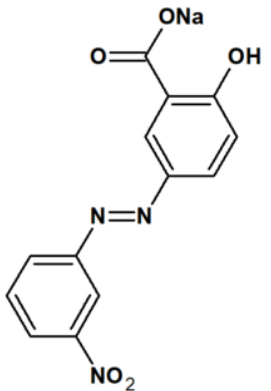

(b)<smiles>O=[N+]([O-])c1ccc(O)cc1</smiles>

(d)

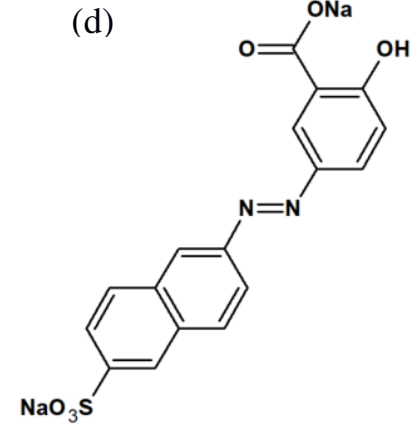

Fig.8 The molecular structures of the LC and the dye molecules. The dyes were dissolved in a surfactant solution. (a) 4-cyano-4'-pentylbiphenyl (5CB) (b) p-nitrophenol (c) alizarin yellow GG (d) chrome yellow. 
255 droplets. The excitation laser has a wavelength of $532 \mathrm{~nm}$ (MLL-III-532) with an intensity of $30 \mathrm{~mW}$.

256 The pendant drop method (DMs-401, Kyowa Kaimen Kagaku) was utilized to monitor the

257 interfacial tension for an LC droplet in a solution. The sample was prepared by preparing a pendant

258 drop $(15 \mu \mathrm{L})$ of $5 \mathrm{CB}$ from a syringe needle into a measurement cell filled with an SDS solution with

259 dye molecules. The image sequence of the pendant drop was acquired by a camera every second, and

260 each interfacial tension was calculated from the droplet shape by fitting with the Young-Laplace

261 equation.

263 Acknowledgments

264 This research was supported by the Institute of Science and Engineering, Chuo University.

266 Author contributions

267 Y. S. and K. K. designed the experiments and analyzed results, and wrote the paper.

269 Competing interests

270 The authors declare no competing interests. 
272 Additional information

273 Supplementary information is available for this paper at ???.

274

275 
276

277

278

279

280

281

282

283

284

285

286

287

288

289

290

291

292

293

294

\section{References}

1. LaMer, V. K. \& Dinegar, R. H. Theory, Production and Mechanism of Formation of Monodispersed Hydrosols. J. Am. Chem. Soc. 72, 4847-4854 (1950).

2. Gao, Z., Rohani, S., Gong, J. \& Wang, J. Recent Developments in the Crystallization Process: Toward the Pharmaceutical Industry. Engineering 3, 343-353 (2017).

3. Yang, Y. \& Nagy, Z. K. Advanced control approaches for combined cooling/antisolvent crystallization in continuous mixed suspension mixed product removal cascade crystallizers. Chemical Engineering Science 127, 362-373 (2015).

4. Alvarez, A. J. \& Myerson, A. S. Continuous Plug Flow Crystallization of Pharmaceutical Compounds. Crystal Growth \& Design 10, 2219-2228 (2010).

5. McGlone, T. et al. Oscillatory Flow Reactors (OFRs) for Continuous Manufacturing and Crystallization. Org. Process Res. Dev. 19, 1186-1202 (2015).

6. Hoshino, M., Khutia, A., Xing, H., Inokuma, Y. \& Fujita, M. The crystalline sponge method updated. IUCrJ 3, 139-151 (2016).

7. Inokuma, Y. et al. X-ray analysis on the nanogram to microgram scale using porous complexes. Nature 495, 461-466 (2013).

8. Mitov, M. Cholesteric liquid crystals in living matter. Soft Matter 13, 4176-4209 (2017).

9. Nersisyan, S. R. \& Tabiryan, N. V. Polarization Imaging Components Based on Patterned Photoalignment. Molecular Crystals and Liquid Crystals 489, 156/[482]-168/[494] (2008). 

axial waveplates for UV-IR wavelengths. Opt. Express, OE 17, 11926-11934 (2009).

11. Wu, H. et al. Arbitrary photo-patterning in liquid crystal alignments using DMD based lithography system. Opt. Express 20, 16684-16689 (2012).

12. Lopez-Leon, T. \& Fernandez-Nieves, A. Drops and shells of liquid crystal. Colloid Polym Sci 289, 345-359 (2011).

13. Wang, X., Miller, D. S., Bukusoglu, E., de Pablo, J. J. \& Abbott, N. L. Topological defects in liquid crystals as templates for molecular self-assembly. Nature Materials 15, 106-112 (2016).

14. Kawaguchi, K., Kageyama, R. \& Sano, M. Topological defects control collective dynamics in neural progenitor cell cultures. Nature 545, 327-331 (2017). 212-216 (2017).

15. Saw, T. B. et al. Topological defects in epithelia govern cell death and extrusion. Nature 544,

17. Milette, J. et al. Reversible long-range patterning of gold nanoparticles by smectic liquid crystals. Soft Matter 8, 6593-6598 (2012). 
19. Pires, D., Fleury, J.-B. \& Galerne, Y. Colloid Particles in the Interaction Field of a Disclination Line in a Nematic Phase. Phys. Rev. Lett. 98, 247801 (2007).

20. Wood, T. A., Lintuvuori, J. S., Schofield, A. B., Marenduzzo, D. \& Poon, W. C. K. A SelfQuenched Defect Glass in a Colloid-Nematic Liquid Crystal Composite. Science 334, 79-83 (2011).

21. Tkalec, U., Ravnik, M., Čopar, S., Žumer, S. \& Muševič, I. Reconfigurable Knots and Links in Chiral Nematic Colloids. Science 333, 62-65 (2011).

22. Jin, C., Krüger, C. \& Maass, C. C. Chemotaxis and autochemotaxis of self-propelling droplet swimmers. PNAS 114, 5089-5094 (2017).

23. Herminghaus, S. et al. Interfacial mechanisms in active emulsions. Soft Matter 10, 7008-7022 (2014).

24. Krüger, C., Bahr, C., Herminghaus, S. \& Maass, C. C. Dimensionality matters in the collective behaviour of active emulsions. Eur. Phys. J. E 39, 64 (2016).

25. Sakai, Y., Sohn, W. Y. \& Katayama, K. Optical motion control of liquid crystalline droplets by host-guest molecular interaction. Soft Matter 15, 7159-7165 (2019).

26. Dogishi, Y., Sakai, Y., Sohn, W. Y. \& Katayama, K. Optically induced motion of liquid crystalline droplets. Soft Matter 14, 8085-8089 (2018).

27. Sakai, Y., Sohn, W. Y. \& Katayama, K. Photo-controllable rotational motion of cholesteric liquid 
crystalline droplets in a dispersion system. RSC Adv. 10, 21191-21197 (2020).

28. Syme, C. D. et al. Frustration of crystallisation by a liquid-crystal phase. Scientific Reports 7, 42439 (2017).

29. Wang, D., Park, S.-Y. \& Kang, I.-K. Liquid crystals: emerging materials for use in real-time detection applications. J. Mater. Chem. C 3, 9038-9047 (2015).

30. Prishchepa, O. O., Shabanov, A. V. \& Zyryanov, V. Y. Director configurations in nematic 031712 (2005).

31. Bushuyev, O. S., Friščić, T. \& Barrett, C. J. Controlling Dichroism of Molecular Crystals by 
345 Figure captions

346 FIG.1 The snapshots of a 5CB droplet in an SDS solution with $p$-nitrophenol $(0.01$ wt $\%)$ under the

347 on-off operation of the UV light is shown; (a) before irradiation, (b) - (e) 0, 30, 60 and 90 s after the

348 UV was turned on, (f) - (h) 0, 60, and $120 \mathrm{~s}$ after the UV light was turned off, (i) and (j) 0 and $30 \mathrm{~s}$

349 after the UV light was turned on again.

350

351 FIG.2 The snapshots of the 5CB droplet in an SDS solution with $p$-nitrophenol $(0.01 \%)$ observed

352 by the polarization microscope under the crossed Nicole condition; (a) before irradiation (b) after the

353 UV irradiation for $60 \mathrm{~s}$. The white arrow in (a) indicates the direction of the analyzer and the polarizer.

FIG. 3 The snapshots of the 5CB droplet in an SDS solution including (a) alizari yellow GG and

(b) chrome yellow under the on-off operation of the UV light is shown; (a) $120 \mathrm{~s}$ after the UV irradiation, (b), (c), and (d) correspond to 120, 240 and $480 \mathrm{~s}$ after the UV was turned on.

FIG. 4 The Raman spectrum of a photo-generated crystal in a 5CB droplet (top). The Raman spectra

for pure 5CB, $p$-nitrophenol (powder), and a $0.3 \mathrm{wt} \%$ SDS solution with $0.01 \mathrm{wt} \%$-nitrophenol are

excitation light source. 
364 FIG. 5 The temporal change of the UV/Vis absorption spectra for the outside SDS solution with $p$ -

365 nitrophenol $(0.001 \%)$. An 5CB droplet was included in the solution, and the outside solution was

366 sampled every 30 second during the UV light irradiation.

367

368 FIG.6 The change of the interfacial tension during the on-off operation of the UV light for a 5CB

369 droplet in a SDS solution with $p$-nitrophenol (0.01 wt\%). The UV light was irradiated twice for 20

370 seconds at 60 and 140 seconds during the measurement.

371

372 FIG 7. The snapshots of the 5CB droplet in an PVA solution with $p$-nitrophenol (0.05 wt $\%)$ under

373 the UV on-off operation is shown. (a) before UV irradiation (b) and (c) $30 \mathrm{~s}, 90 \mathrm{~s}$ after the UV light

374 turned on, (d) $60 \mathrm{~s}$ after the UV light turned off.

FIG 8. The molecular structures of the LC and the dye molecules. The dyes were dissolved in a

surfactant solution. (a) 4-cyano-4'-pentylbiphenyl (5CB) (b) p-nitrophenol (c) alizarin yellow GG (d) 


\section{Figures}

(a)

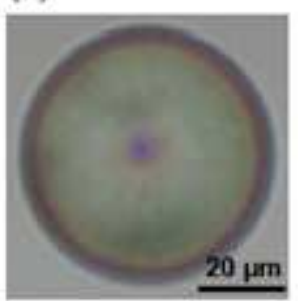

(f)

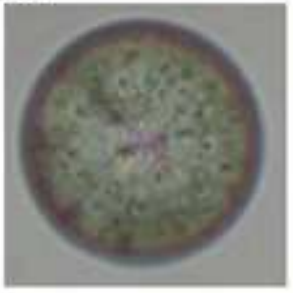

(b)

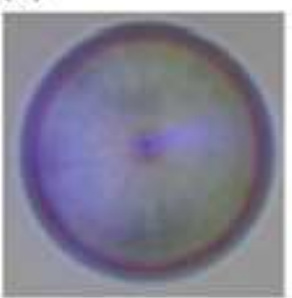

(g)

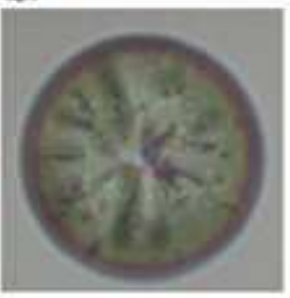

(c)

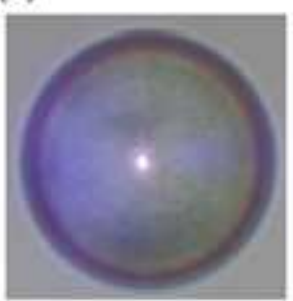

(h)

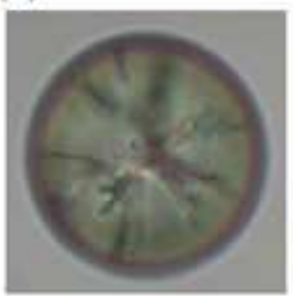

(d)

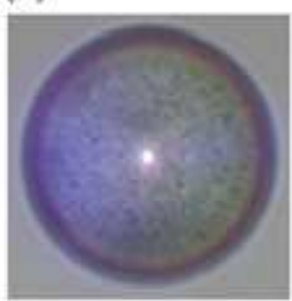

(i)

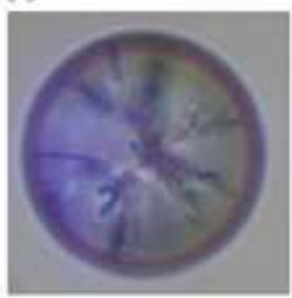

(e)

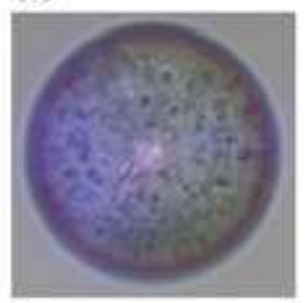

(j)

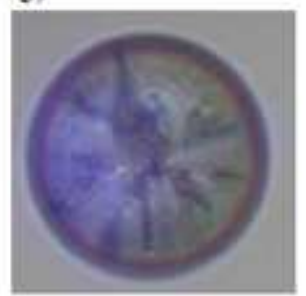

\section{Figure 1}

The snapshots of a 5CB droplet in an SDS solution with p-nitrophenol $(0.01 \mathrm{wt} \%)$ under the on-off operation of the UV light is shown; (a) before irradiation, (b) - (e) 0, 30, 60 and $90 \mathrm{~s}$ after the UV was turned on, (f) - (h) 0,60, and $120 \mathrm{~s}$ after the UV light was turned off, (i) and (j) 0 and $30 \mathrm{~s}$ after the UV light was turned on again.

\section{(a)}

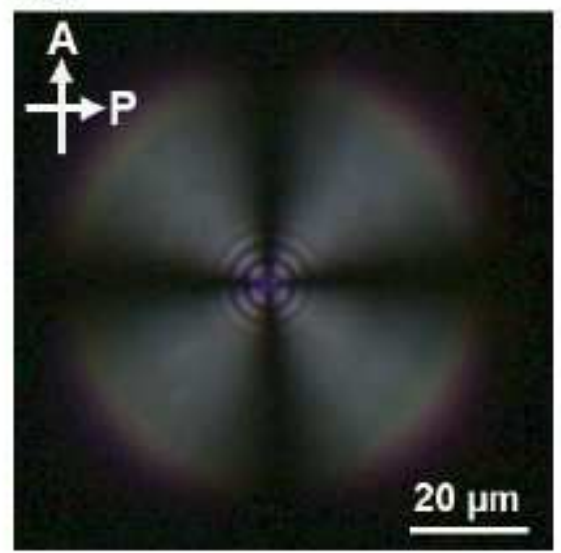

(b)

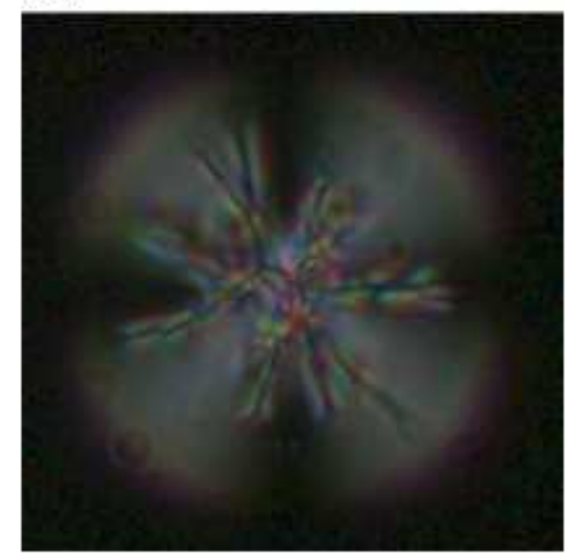

\section{Figure 2}

The snapshots of the 5CB droplet in an SDS solution with p-nitrophenol $(0.01 \%)$ observed by the polarization microscope under the crossed Nicole condition; (a) before irradiation (b) after the UV irradiation for $60 \mathrm{~s}$. The white arrow in (a) indicates the direction of the analyzer and the polarizer. 
(a)
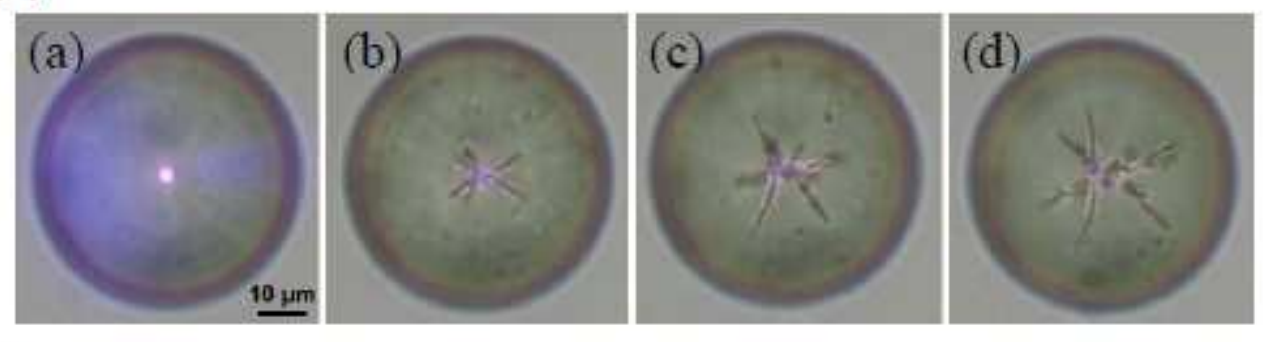

(b)
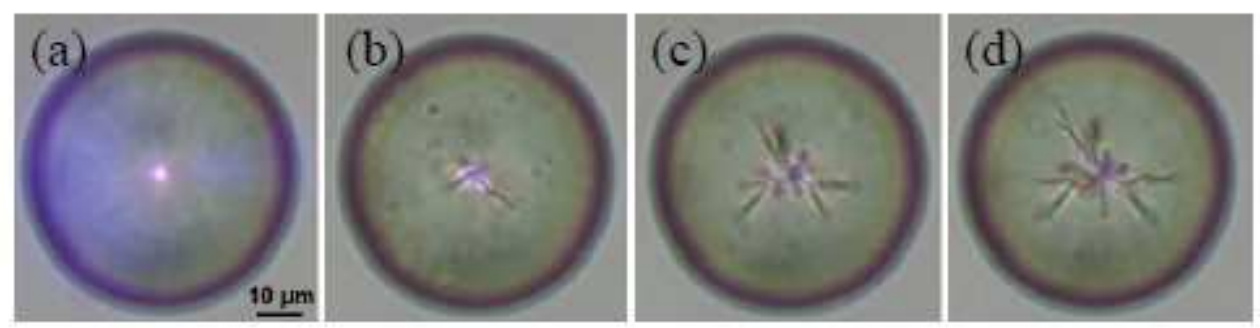

\section{Figure 3}

The snapshots of the 5CB droplet in an SDS solution including (a) alizari yellow GG and (b) chrome yellow under the on-off operation of the UV light is shown; (a) $120 \mathrm{~s}$ after the UV irradiation, (b), (c), and (d) correspond to 120,240 and $480 \mathrm{~s}$ after the UV was turned on.

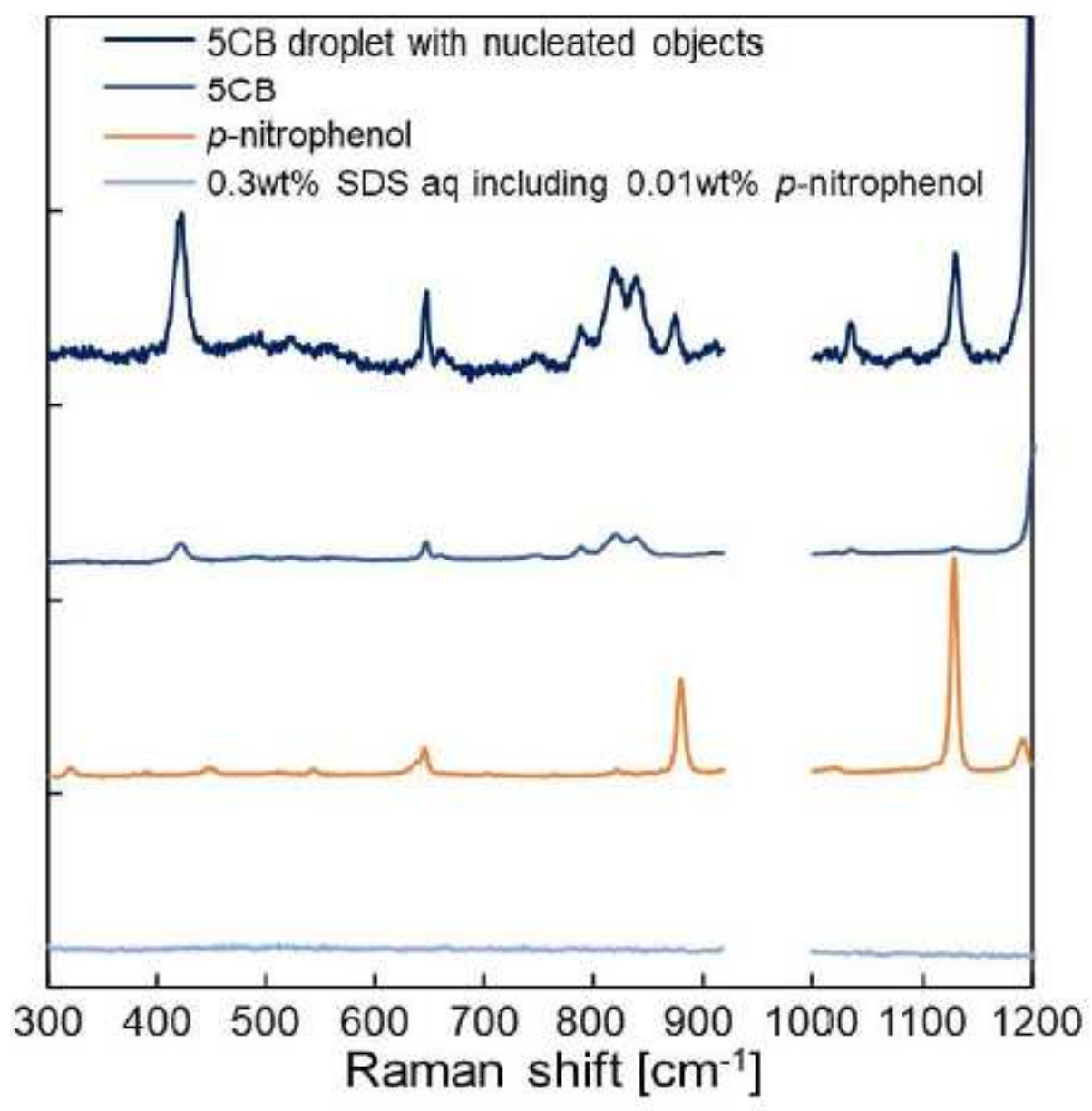


Figure 4

The Raman spectrum of a photo-generated crystal in a 5CB droplet (top). The Raman spectra for pure 5CB, p-nitrophenol (powder), and a 0.3wt\% SDS solution with $0.01 \mathrm{wt} \% \mathrm{p}$-nitrophenol are shown for comparison. The spectral region from 920-1000 cm-1 was removed due to the noise of the excitation light source.

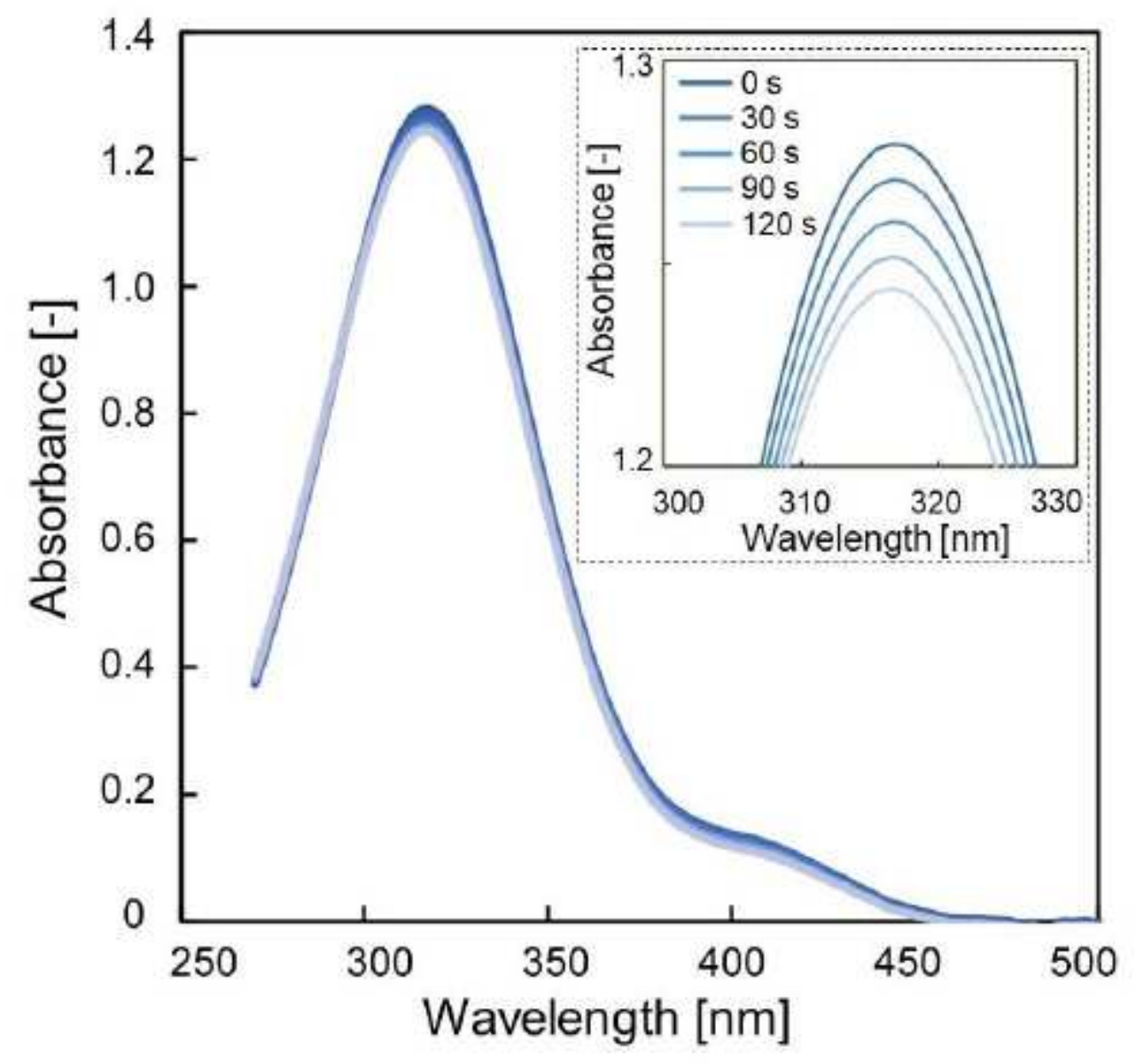

\section{Figure 5}

The temporal change of the UV/Vis absorption spectra for the outside SDS solution with p- nitrophenol $(0.001 \%)$. An 5CB droplet was included in the solution, and the outside solution was sampled every 30 second during the UV light irradiation. 


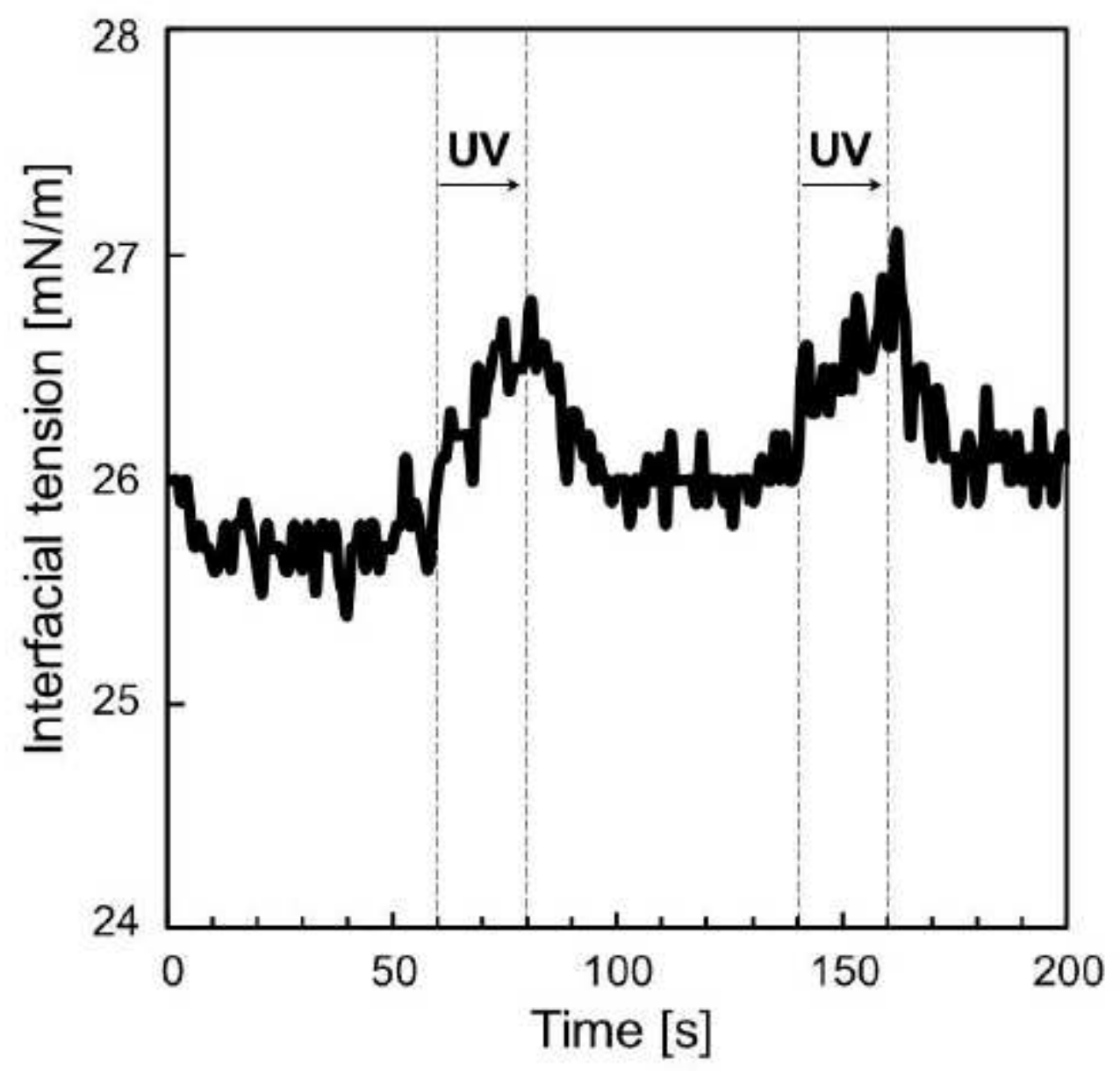

Figure 6

The change of the interfacial tension during the on-off operation of the UV light for a 5CB droplet in a SDS solution with p-nitrophenol ( $0.01 \mathrm{wt} \%)$. The UV light was irradiated twice for 20 seconds at 60 and 140 seconds during the measurement.
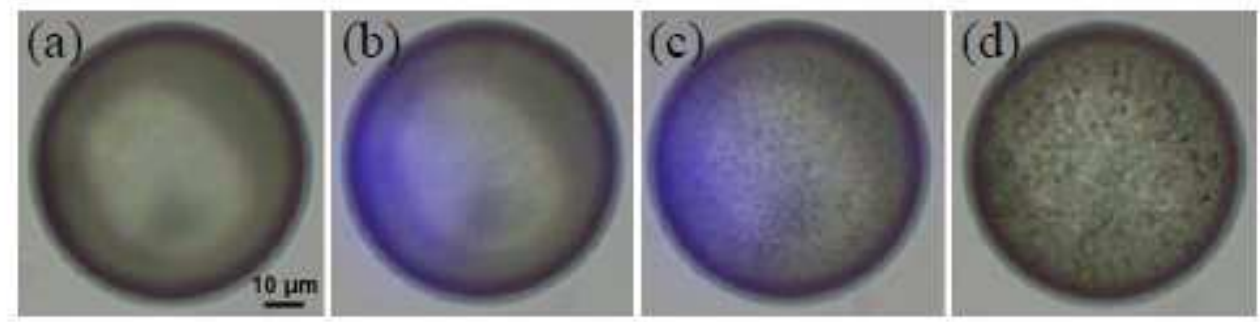

Figure 7

The snapshots of the 5 CB droplet in an PVA solution with p-nitrophenol $(0.05 \mathrm{wt} \%)$ under the UV on-off operation is shown. (a) before UV irradiation (b) and (c) $30 \mathrm{~s}, 90 \mathrm{~s}$ after the UV light turned on, (d) $60 \mathrm{~s}$ after the UV light turned off. 


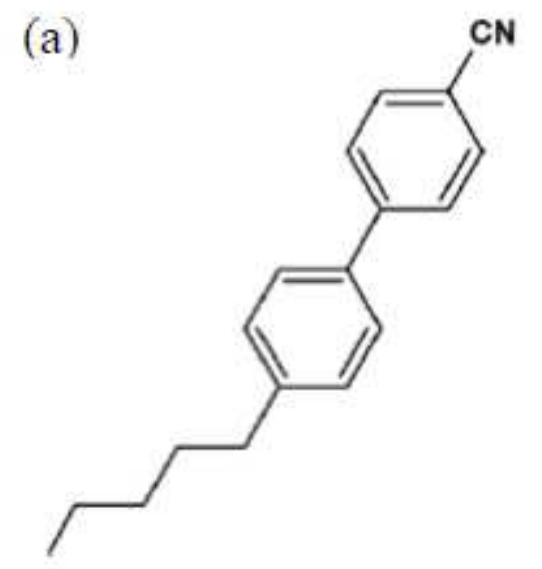

(b)
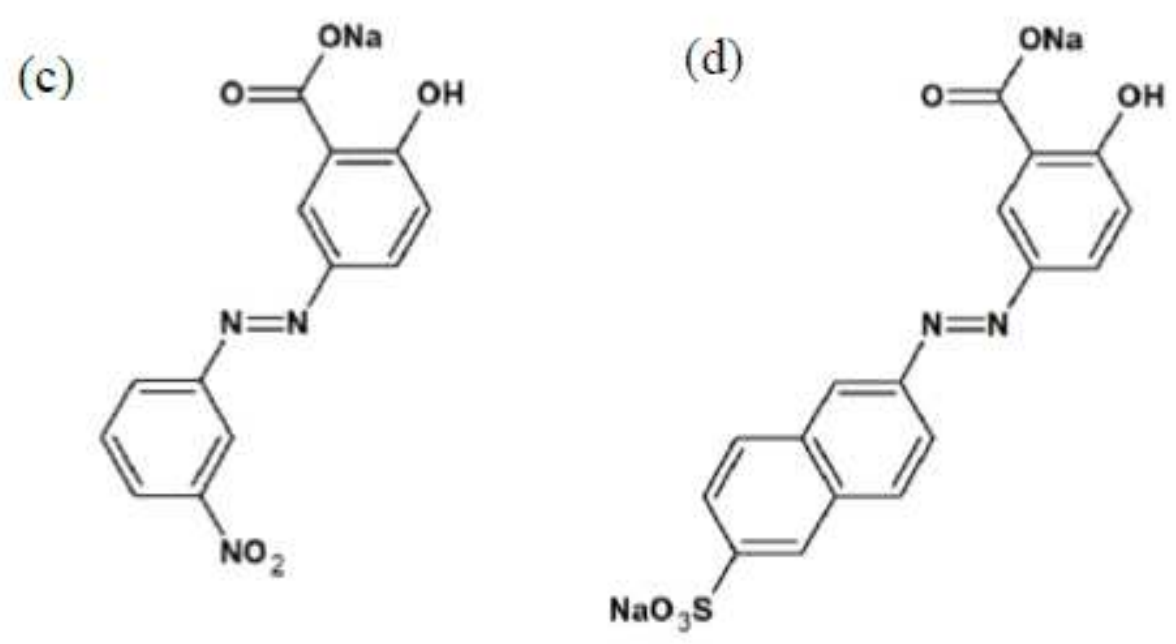

\section{Figure 8}

The molecular structures of the LC and the dye molecules. The dyes were dissolved in a surfactant solution. (a) 4-cyano-4'-pentylbiphenyl (5CB) (b) p-nitrophenol (c) alizarin yellow GG (d) chrome yellow

\section{Supplementary Files}

This is a list of supplementary files associated with this preprint. Click to download.

- 20210215Supportinginformation.pdf

- MovieS45CBdropletPVApnitrophenol.mp4

- MovieS3a5CBdropletSDSalizarinyellow.mp4

- MovieS3b5CBdropletSDSchromeyellow.mp4

- MovieS65CBdropletSDSpnitorphenolheater.mp4

- MovieS5toluenedropletSDSpnitrophenol.mp4

- MovieS25CBdropletSDSpnitrophenolcrossnicole.mp4 
- MovieS15CBdropletSDSpnitrophenol.mp4 